\title{
Semiautomated Volumetric Measurement on Postcontrast MR Imaging for Analysis of Recurrent and Residual Disease in Glioblastoma Multiforme
}

\author{
D.S. Chow, J. Qi, X. Guo, V.Z. Miloushev, F.M. Iwamoto, J.N. Bruce, A.B. Lassman, L.H. Schwartz, A. Lignelli, B. Zhao, and C.G. Filippi
}

\begin{abstract}
BACKGROUND AND PURPOSE: A limitation in postoperative monitoring of patients with glioblastoma is the lack of objective measures to quantify residual and recurrent disease. Automated computer-assisted volumetric analysis of contrast-enhancing tissue represents a potential tool to aid the radiologist in following these patients. In this study, we hypothesize that computer-assisted volumetry will show increased precision and speed over conventional $1 \mathrm{D}$ and 2D techniques in assessing residual and/or recurrent tumor.
\end{abstract}

MATERIALS AND METHODS: This retrospective study included patients with native glioblastomas with MR imaging performed at $24-48$ hours following resection and 2-4 months postoperatively. ID and 2D measurements were performed by 2 neuroradiologists with Certificates of Added Qualification. Volumetry was performed by using manual segmentation and computer-assisted volumetry, which combines region-based active contours and a level set approach. Tumor response was assessed by using established 1D, 2D, and volumetric standards. Manual and computer-assisted volumetry segmentation times were compared. Interobserver correlation was determined among ID, 2D, and volumetric techniques.

RESULTS: Twenty-nine patients were analyzed. Discrepancy in disease status between ID and 2D compared with computer-assisted volumetry was $10.3 \%(3 / 29)$ and $17.2 \%(5 / 29)$, respectively. The mean time for segmentation between manual and computer-assisted volumetry techniques was 9.7 minutes and $<1$ minute, respectively $(P<.01)$. Interobserver correlation was highest for volumetric measurements $(0.995 ; 95 \% \mathrm{Cl}$, $0.990-0.997)$ compared with $1 \mathrm{D}(0.826 ; 95 \% \mathrm{Cl}, 0.695-0.904)$ and $2 \mathrm{D}(0.905 ; 95 \% \mathrm{Cl}, 0.828-0.948)$ measurements.

CONCLUSIONS: Computer-assisted volumetry provides a reproducible and faster volumetric assessment of enhancing tumor burden, which has implications for monitoring disease progression and quantification of tumor burden in treatment trials.

ABBREVIATIONS: $C A V=$ computer-assisted volumetry; GBM = glioblastoma multiforme; RANO = Response Assessment in Neuro-Oncology

G lioblastoma multiforme (GBM) is an invasive and highly aggressive tumor with a median patient survival of 14.6 months with combined radiation therapy and temozolomide. ${ }^{1}$ New therapies are being developed to treat GBM, which may decrease morbidity and lengthen the period of progression-free survival. However, to fully use new therapies in the treatment of GBM, quantitative MR imaging metrics are needed to guide therapy, risk stratify patients undergoing therapy, and prognosticate outcome. ${ }^{2,3}$ A major limitation is this lack of prognostic imaging parameters. ${ }^{4}$ Simple radio-

Received April 14, 2013; accepted after revision June 22.

From the Departments of Radiology (D.S.C., J.Q., X.G., V.Z.M., L.H.S., A.L., B.Z., C.G.F.), Neurology (F.M.I., A.B.L.), and Neurosurgery (J.N.B.), College of Physicians and Surgeons, Columbia University, New York, New York.

Author contributions: guarantors of integrity of entire study: D.S.C., C.G.F.; study concepts/study design: D.S.C., B.Z., A.L., C.G.F.; study data acquisition or data analysis/ interpretation: D.S.C., J.Q., X.G., V.Z.M., A.L., B.Z., C.G.F.; manuscript drafting or manuscript revision for important intellectual content: D.S.C., J.Q., X.G., V.Z.M., F.M.I., J.N.B., A.B.L., L.H.S., A.L., B.Z., C.G.F.; manuscript editing: D.S.C., L.H.S., B.Z., C.G.F.; literature research: D.S.C., L.H.S., B.Z., C.G.F.; approval of the final version of the submitted manuscript: D.S.C., J.Q., X.G., V.Z.M., F.M.I, J.N.B, A.B.L, L.H.S., A.L., B.Z., C.G.F. graphic monitoring with freehand measurements of the amount of contrast-enhancing tumor in 2 or 3 planes is commonly used for assessing response to different therapies, which is used to guide treatment strategies. ${ }^{5,6}$ Commonly used techniques include the Response Evaluation Criteria in Solid Tumors and the MacDonald criteria, which use unidimensional and bidimensional measurements, respectively. ${ }^{7-9}$ However, the postsurgical cavity tends to be highly irregular in shape, which may increase the difficulty in obtaining accurate and reproducible measurements. In particular, singledimensional techniques may be inaccurate, given the propensity of high-grade gliomas to grow in an eccentric and nodular fashion, and may not be reflective of change in actual tumor burden. ${ }^{10}$

Paper previously presented in part at: 51st Annual Meeting of the American Society of Neuroradiology, May 18-23, 2013; San Diego, California, under the title "Semi-Automated Measurement of Recurrent and Residual Disease in Glioblastoma Multiforme: A Potential New Objective Metric."

Please address correspondence to Daniel S. Chow, MD, College of Physicians and Surgeons, Columbia University Medical Center, 180 Fort Washington Ave, HP-3-311, New York, NY; e-mail: dsc9004@nyp.org

http://dx.doi.org/10.3174/ajnr.A3724 
Recently, the Response Assessment in Neuro-Oncology (RANO) Working Group proposed new recommendations for assessing response criteria for high-grade gliomas, which included a modification to the MacDonald criteria. ${ }^{11}$ While the RANO criterion used 2D measurements, the Working Group suggested that volumetric analysis could provide more accurate measurements with respect to bidimensional techniques. ${ }^{11}$ Outside the central nervous system, volumetric assessment has been proved superior to unidimensional measurements when used to assess treatment response in hepatic, pulmonary, and pancreatic malignancies. ${ }^{12,13}$

Despite the potential advantages of volumetric assessment, this technique requires manual outlining of the contrast-enhancing border, which can be both time-consuming and technically challenging. ${ }^{5,10}$ This technique may be further limited in cases with irregular enhancement and subependymal extension. ${ }^{10}$ For these reasons, computer-aided volumetry techniques applied to the contrast-enhancing tissue represent a potential tool to aid the radiologist in following these patients. Such techniques may increase both the accuracy and reproducibility in assessing GBM recurrence. Thus far, such automation has been explored in the assessment of advanced lung cancer with promising results. ${ }^{14} \mathrm{Ap}-$ plication of computer-assisted volumetry (CAV) has been explored for the evaluation of gliomas; however, these studies have dealt with native nontreated disease and have not been validated against other measurement techniques. ${ }^{15-17}$

In this study, we describe a novel CAV technique for assessment of tumor burden in the patient with GBM. Specifically, we describe the reliability and feasibility of this technique compared with traditional linear-based measurements in the patient with postresection GBM.

\section{MATERIALS AND METHODS \\ Subjects}

After institutional review board approval of this Health Insurance Portability and Accountability Act-complaint study, a query of the neuropathology department data base at our institution from January 2011 to November 2012 was conducted. Specifically, patients who had undergone primary resection of glioblastomas were evaluated. Given that many of our patients are referred from other facilities for resections, we specifically examined patients who had undergone MR imaging evaluation at our institution where standardization of MR imaging protocols allows a better comparison of scans. A retrospective review of these medical records was conducted to determine demographic information including sex, age at time of resection, extent of resection, and duration between follow-up imaging examinations. All patients received combination radiation therapy with concurrent chemotherapy following maximal resection.

\section{Imaging Techniques}

All imaging was performed on a 3T MR imaging system (Signa; GE Healthcare, Milwaukee, Wisconsin) by using an 8-channel head-array coil (Signa HDxt; GE Healthcare). We obtained the following sequences: axial T1-weighted pre- and postcontrast, T2, and FLAIR. Volumetric acquisitions were also acquired for all postcontrast images with a T1-weighted 3D inversion recovery fast spoiled gradient-recalled sequence with the following parameters: TI, $450 \mathrm{~ms}$; TR, $10.2 \mathrm{~ms}$; TE, $4.2 \mathrm{~ms} ; \alpha$, 13; bandwidth, 25
$\mathrm{KHz}$; FOV, $25 \mathrm{~cm}$; matrix, $256 \times 256$; section thickness, $1.2 \mathrm{~mm}$. Total scanning time was approximately 4 minutes 15 seconds. Postcontrast images were acquired by using intravenous gadobenate dimeglumine (MultiHance; Bracco, Milan, Italy) with a weight-based dose of $0.2 \mathrm{~mL} / \mathrm{kg}$. The time between intravenous injection and postcontrast imaging was 5 minutes. All immediate postoperative imaging was performed within 2 days of resection by using the same MR imaging parameters. Follow-up imaging was performed between 2 and 4 months after surgery.

\section{Imaging Interpretation}

Two radiologists (C.G.F. and A.B.L.), who were blinded to the final calculated volume, each measured the major and minor axes for each tumor on postcontrast sequences. The major axis was defined as the longest diameter, and the minor axis was defined as the longest diameter perpendicular to the major axis. Gross total resection was defined as the absence of enhancing tissue at postoperative examination. Nonmeasurable lesions were defined as enhancing masses with lesions with maximal thickness of $<4 \mathrm{~mm}$ (2 times the imaging section thickness) to reduce variability from volume averaging. ${ }^{11}$ Additionally, the surgical cavity, cysts, and necrosis were not included in measurements per the RANO criteria. ${ }^{11}$ In cases of multiple enhancing foci, individual diameters were measured and summed for response evaluation. ${ }^{10,11}$

Manual volumetric segmentations were performed by 1 radiologist (D.S.C.), who was blinded to the final CAV calculation, by performing manual tracing around the enhancing lesion on volumetric sequences (section thickness, $0.2 \mathrm{~mm}$ ). All manual segmentations were performed at a dedicated workstation (Advantage Workstation, Version 4.3; GE Healthcare Europe, Buc, France). All measurements were performed by comparison with precontrast T1 images to avoid T1 shortening effects from postsurgical changes (ie, blood products). The time required to perform manual segmentation was also recorded.

CAV measurements were performed by a separate radiologist (J.Q.) who was blinded to final manual volumetric measurements. Tumor contours were delineated on immediate and 2- to 4-month postsurgery $\mathrm{T} 1$-weighted MR images by using an in-house, proprietary segmentation algorithm developed to assist the computer-aided volume calculation of this project. Our CAV algorithm was originally developed for hepatic lesions and has since been adapted for different applications including brain and renal malignancies, lymphoma, and peritoneal mesothelioma. ${ }^{18-22}$ The semiautomated algorithm combines the region-based active contours and a level set approach and has the advantages of easy initialization, quick segmentation, and efficient modification. An operator manually selects a region of interest that roughly encloses the tumor on a single image. Boundary localization of the tumor and exclusion of the nonviable tissue inside the tumor are then performed automatically by the developed algorithm. Once the segmentation is completed on an image, the tumor contour is propagated to its neighboring images, serving as an initial region of interest for subsequent segmentations on the neighboring images. This process continues iteratively until all the tumor images are segmented. To ensure correct results, computer-generated tumor contours are superimposed on the original images for inspection and modification as needed by a radiologist. Once the segmentation is finalized, tumor volume is automatically calculated (Fig 1). This seg- 
mentation algorithm and a number of manual interaction functions, such as selection of a region of interest and modification of suboptimal contour results, have been integrated into a user-friendly imageviewing system developed with the Matlab (MathWorks, Natick, Massachusetts) computer language by the research group.

\section{Definition of Response}

Tumor response was assessed for each study by using established 1D, $2 \mathrm{D}$ and volumetric standards extrapolated from bi-dimensional standards. ${ }^{7-9}$ Cases of discrepancy between readers were re-reviewed to reach a consensus. Volumetric standards were established by using an extrapolation from the MacDonald criteria by converting orthogonal measurements to a volume assuming a spheric lesion (Table 1). ${ }^{10}$ "Complete response" was defined as complete absence of contrast-enhancing tumor for all techniques. "Partial response" was defined as $>30 \%$ decrease in the sum of maximal diameters for $1 \mathrm{D}$, $>50 \%$ decrease in the product of orthogonal diameters for $2 \mathrm{D}$, and $>65 \%$ in volume for volumetric techniques. "Progression of disease" was defined as a $20 \%$ increase in the sum of maximal diameters for $1 \mathrm{D},>25 \%$ increase in the product of orthogonal diameters for $2 \mathrm{D}$, and $>40 \%$ increase in volume for volumetric techniques. "Stable disease" was defined as all other changes for 1D, 2D, and volumetric techniques. Tumor response counts and discrepancy between agreements were calculated among the different measuring methods.

\section{Statistical Tests}

Manual and semiautomated volumetric measurements and segmentation times were compared by using a paired $t$ test. Pearson correlation coefficients with corresponding confidence intervals were used to assess interobserver correlations of $1 \mathrm{D}, 2 \mathrm{D}$, and volumetric measurements. The inter-rater agreement statistic with
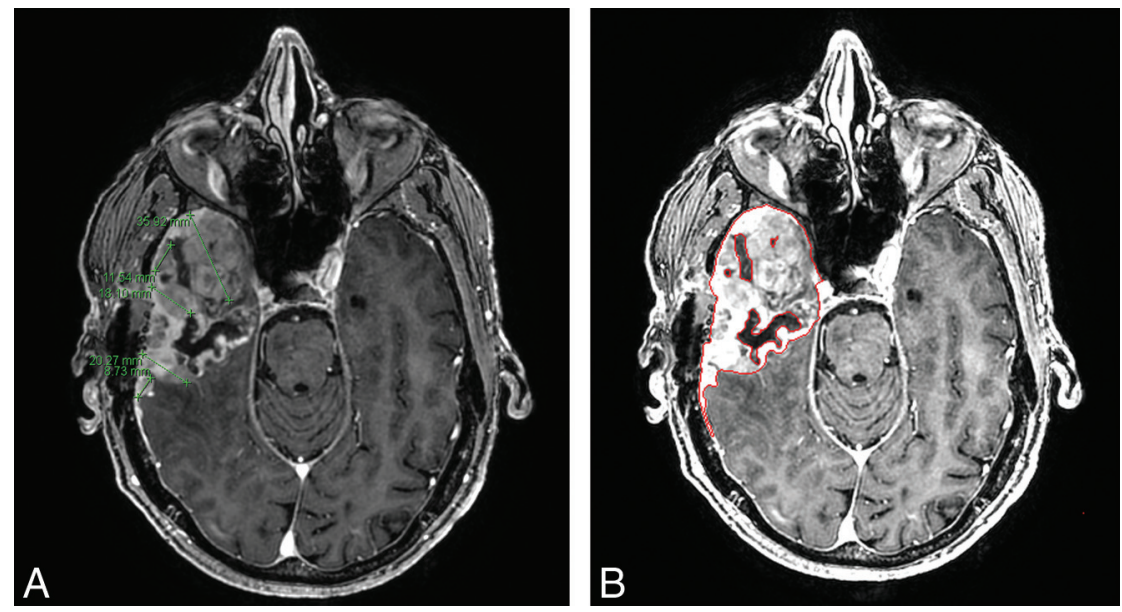

FIG 1. Comparison of linear ID measurements $(A)$ and $C A V$ analysis $(B)$ in a 64-year-old man with glioblastoma multiforme 11 weeks following resection. Our readers found volumetric analysis preferable, given the irregularity of recurrence. corresponding confidence intervals was calculated for tumor response by using weighted $\kappa$ values. $\kappa$ values were interpreted as follows: $0.40-0.60$, moderate; $0.61-0.80$, good; and 0.81-1.00, very good. Statistical analysis was conducted with MedCalc for Windows, Version 12.2.1 (MedCalc Software, Mariakerke, Belgium). A $P$ value $<.05$ was considered statistically significant.

\section{RESULTS}

\section{Subjects}

In total, we identified 90 patients who had a GBM resection at our institution between January 2011 and November 2012, of whom $32.2 \%$ (29/90) had undergone all standardized postoperative MR imaging at our institution. The mean age of patients was $62.2 \pm 8.5$ years (range, $38-81$ years); of whom $55 \%(16 / 29)$ were men and $45 \%(13 / 29)$ were women. With respect to the extent of resection, $5 / 29$ and 24/29 underwent gross total resection and subtotal resection, respectively. Demographic information is summarized in Table 2. The mean time to follow-up from baseline postoperative imaging was $12.3 \pm 3$ weeks (range, $6-16$ weeks).

\section{Disease Response}

Discrepancies in disease response for $1 \mathrm{D}, 2 \mathrm{D}$, and volumetric techniques were encountered in $17.2 \%$ (5/29), 10.3\% (3/29), and 3.4\% $(1 / 29)$ of patients. Following review of discrepancies, the complete response rate was 3.4\% (1/29) for all measuring techniques. The partial response rate was $10.3 \%(3 / 29), 10.3 \%(3 / 29)$, and 6.9\% (2/29) for $1 \mathrm{D}, 2 \mathrm{D}$, and CAV techniques, respectively. The stable disease rate was $10.3 \%(3 / 29), 10.3 \%(3 / 29)$, and $17.2 \%(5 / 29)$ for $1 \mathrm{D}, 2 \mathrm{D}$, and CAV techniques, respectively. The progressive disease rate was $75.9 \%$ (22/29), 75.9\% (22/29), and 72.4\% (21/29) for 1D, 2D, and CAV techniques, respectively. These results are summarized in Table 3. Discrepancies in disease classification between $1 \mathrm{D}$ and 2D compared with CAV were observed in $10.3 \%(3 / 29)$ and $17.2 \%(5 / 29)$ of cases (Figs 2 and 3). The discrepancy between $1 \mathrm{D}$ and $2 \mathrm{D}$ was $6.8 \%(2 / 29)$.

\section{Analysis of Measurement Techniques}

The mean volume for manual and CAV analyses was 10.0 and $9.5 \mathrm{~mL}$, respectively $(P=.11)$. The mean time for segmentation between manual and CAV techniques was 9.7 minutes and $<1$ minute, respectively $(P<.01)$. The Pearson correlation between manual and CAV analysis was 0.995 (95\% CI, 0.990-0.997). This correlation was significantly higher than interobserver correlations for 1D (Pearson correlation, 0.826; 95\% CI, 0.695-0.904; $P<.0001)$ and 2D

\section{Table 1: Definition of response criteria for unidimensional (RECIST), bidimensional (Macdonald), and volumetric techniques}

\begin{tabular}{|c|c|c|c|c|}
\hline Criteria & CR & PR & SD & PD \\
\hline RECIST & Resolution of all enhancement & $\begin{array}{l}\geq 30 \% \text { Decrease in sum of } \max \\
\text { dimensions }\end{array}$ & All others & $\begin{array}{l}\geq 20 \% \text { Increase in sum of } \max \\
\text { dimensions }\end{array}$ \\
\hline Macdonald & Resolution of all enhancement & $\begin{array}{l}\geq 50 \% \text { Decrease in product of } 2 \\
\text { orthogonal dimensions }\end{array}$ & All others & $\begin{array}{l}\geq 25 \% \text { Increase in product of } 2 \\
\text { orthogonal dimensions }\end{array}$ \\
\hline Volumetric & Resolution of all enhancement & $\geq 65 \%$ Decrease in volume & All others & $\geq 40 \%$ Increase in volume \\
\hline
\end{tabular}

Note:-CR indicates complete response; PR, partial response; SD, stable disease; PD, progression of disease; RECIST, Response Evaluation Criteria in Solid Tumors; max, maximal. 
(Pearson correlation, 0.905; 95\% CI, 0.828-0.948; $P<.0001$ ) measurements (Table 4). No significant difference was observed between $1 \mathrm{D}$ and $2 \mathrm{D}$ correlations $(P=.12)$. With regard to classifying disease response, inter-reader agreement was significantly higher for volumetric techniques $(\kappa=0.948 ; 95 \%$ CI, $0.845-1.000)$ compared with $1 \mathrm{D}(P=.0002 ; \kappa=0.760 ; 95 \% \mathrm{CI}, 0.682-1.000)$ and $2 \mathrm{D}(P=.01$;

\section{Table 2: Demographic information of included patients}

\begin{tabular}{lc}
\hline \multicolumn{1}{c}{ Variable } & Value \\
\hline Male/female (No.) & $16: 13$ \\
Mean age at resection (yr, SD) & $62.2(8.5)$ \\
Mean follow-up (wk, SD) & $12.3(3.0)$ \\
Extent of resection (No., \%) & \\
$\quad$ GTR & $5(17.2)$ \\
STR & $24(82.8)$ \\
\hline
\end{tabular}

Note:-GTR indicates gross total resection; STR, subtotal resection.

Table 3: Response counts of 1D, 2D, and CAV $(n=29)$

\begin{tabular}{lcccc}
\hline & CR & PR & SD & PD \\
\hline ID & 1 & 3 & 3 & 22 \\
2D & 1 & 4 & 2 & 22 \\
CAV & 1 & 2 & 5 & 21 \\
\hline
\end{tabular}

Note:-CR indicates complete response; PR, partial response; SD, stable disease; PD, progression of disease.
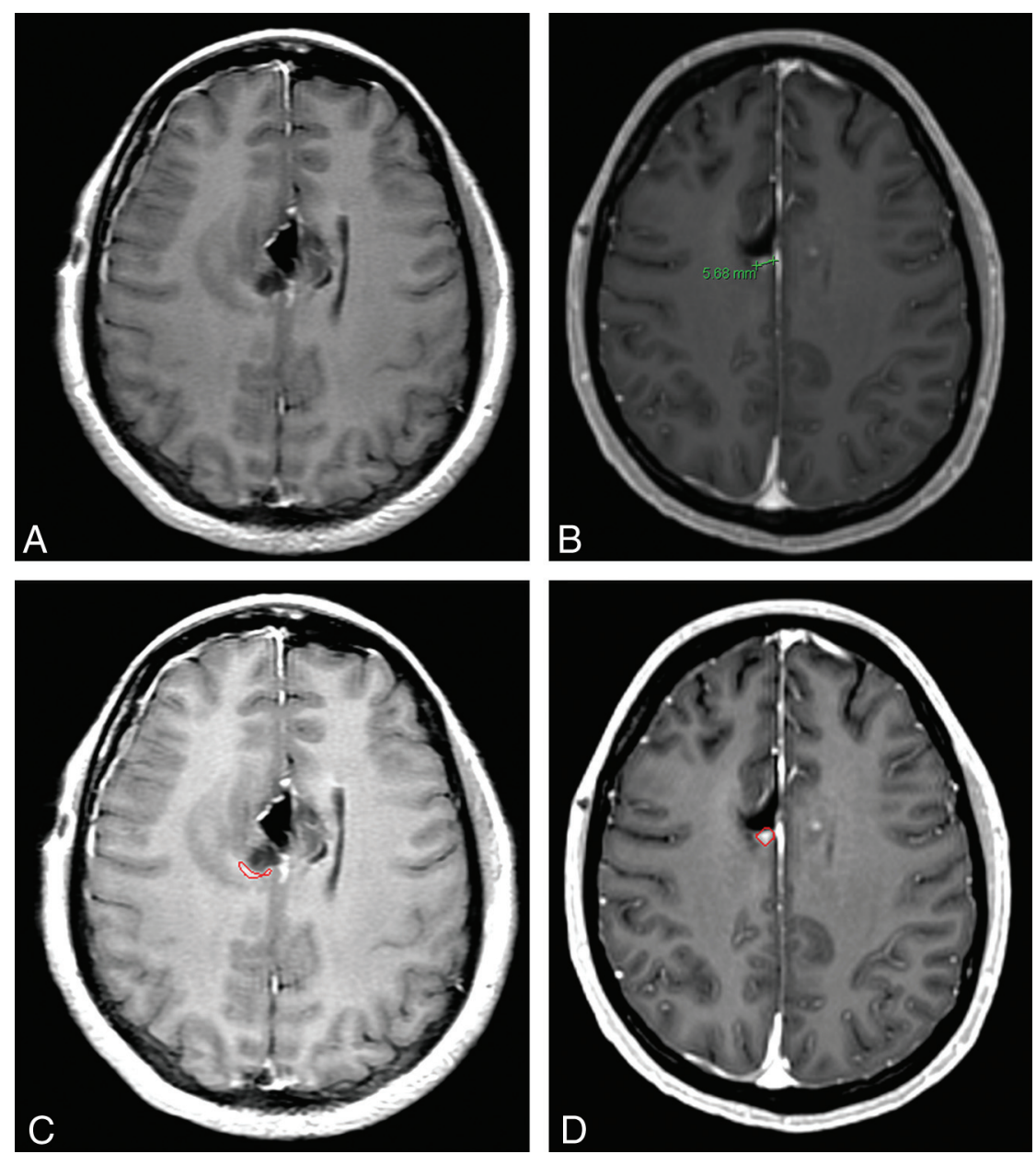

FIG 2. Disease status categorization in a 38-year-old woman with glioblastoma following resection, at 24 hours and at 12 weeks. ID measurement found the contrast enhancement on initial postoperative imaging nonmeasurable $(A)$ and subsequently labeled this case disease progression on follow-up imaging $(B)$. CAV measurement labeled this case stable disease between baseline $(C)$ and follow-up imaging $(D)$. $\kappa=0.851 ; 95 \%$ CI, 0.555-0.966) measurements (Table 5). No significant difference was observed between $1 \mathrm{D}$ and $2 \mathrm{D}$ agreements $(P=.25)$.

\section{DISCUSSION}

Surveillance and management of patients with GBM are reliant on imaging; however, measurement of residual disease can be challenging. In the present study, we examined a CAV approach to quantify residual disease and noted no significant difference compared with manual volumetric measurements, which are time-consuming and impractical in a busy clinical or academic practice. Additionally, CAV assessment was least variable compared with unidimensional and bidimensional techniques. These results are in line with other studies comparing manual volumetric tracing with diameter-based approaches, which have also noted less inter-reader and intrareader variability for volumetric techniques. ${ }^{23}$ While volumetric analysis is technically challenging and time-consuming, the CAV process that we have developed was both significantly faster and reliable, making application for routine clinical use and investigative purposes feasible. Other studies have described similar computer-aided techniques in the evaluation of gliomas; however, validation against other measurement techniques is lacking. ${ }^{15}$

Bidimensional techniques used in the MacDonald and RANO criteria are currently the most commonly used techniques for GBM assessment; however, studies have reported high interobserver and intraobserver variability, even among individuals with specialty training in neuroradiology. ${ }^{24,25}$ The increased variability in diameter-based approaches likely stems from the inherent irregularities in GBM. Specifically, GBMs are not solid ellipsoid lesions but instead typically display complex morphology with infiltrative margins and eccentric growth and demonstrate heterogeneity with areas of cystic degeneration and necrosis. Additionally, linear measurements may be affected by differences in head positioning at the time of examination and scan section techniques. These limitations are further compounded in the postoperative patient in whom blood products may be mistaken for enhancing tumor and the surgical cavity itself may have collapsed. ${ }^{26}$

Assessing residual volume in patients with GBM has been shown to be a significant and independent predictor. ${ }^{4,27,28}$ Intuitively, 3D volumetric analysis is a more accurate method for assessing tumor size compared with alternative linear-based techniques. $^{23,26,29}$ Dempsey et $\mathrm{al}^{5}$ found volumetric analysis to be predictive of survival compared with diameter-based analysis, which failed to adequately assess tumor size in recurrent gliomas. Such volumetric 

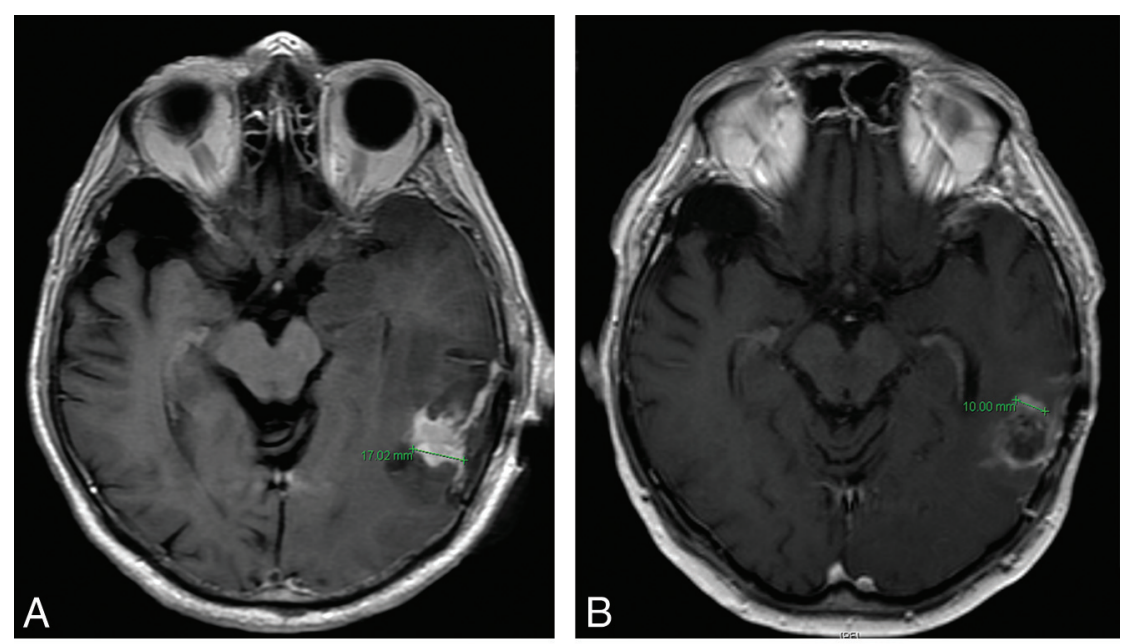

Accurate assessment of tumor volume is important for clinical management and particularly in the development of new therapies and trials. This is of particular importance in the evaluation of GBM, given its exceedingly aggressive behavior. ${ }^{30}$ When assessing clinical response determined by different models, we observed a $17.2 \%$ difference in outcome classification when comparing volumetric with bidimensional techniques; however, similar studies have observed up to a $40 \%$ discrepancy. ${ }^{24}$ Additionally, disease status categorizations are defined on thresholds based on presumed ellipsoid geometry, which may, in turn, lead to increased variability in assessing GBM,
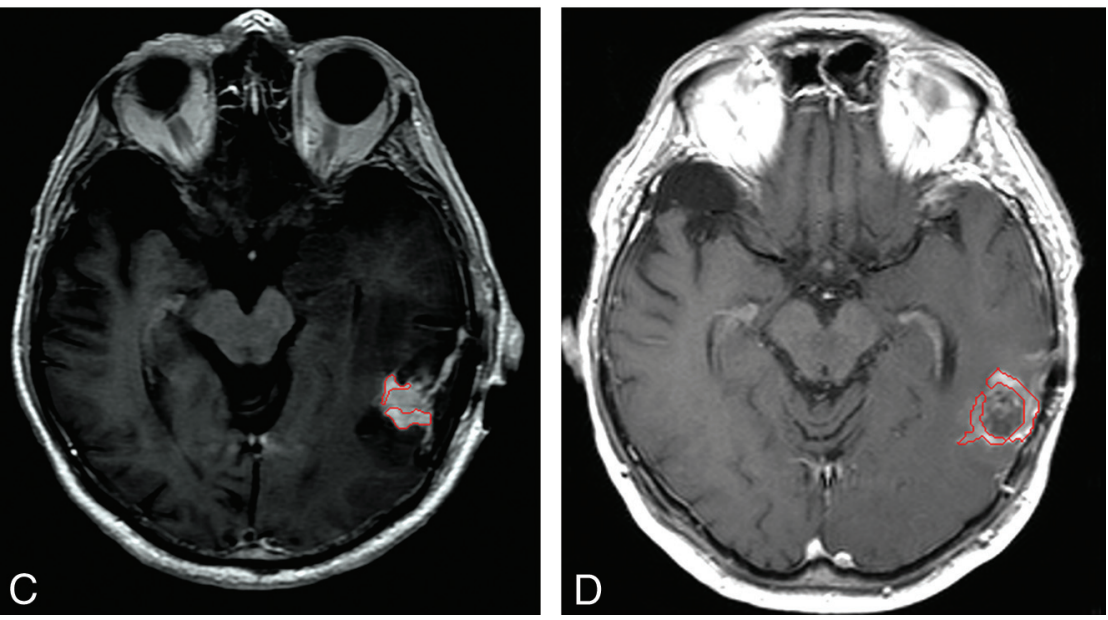

FIG 3. Disease status categorization in an 81-year-old man with glioblastoma following resection at 24 hours and at 12 weeks. ID measurement labeled this case partial response between baseline $(A)$ and follow-up imaging (B). CAV measurement labeled this case stable disease between baseline $(C)$ and follow-up imaging $(D)$.

Table 4: Interobserver correlation ${ }^{\mathrm{a}}$ of 1D, 2D, and volumetric ${ }^{\mathrm{b}}$ measurements

\begin{tabular}{lccc}
\hline & \multirow{2}{*}{$\begin{array}{c}\text { Interobserver Pearson } \\
\text { Correlation }\end{array}$} & \multicolumn{2}{c}{$95 \% \mathrm{Cl}$} \\
\cline { 3 - 4 } & 0.814 & Lower Limit & Upper Limit \\
\hline ID & 0.904 & 0.686 & 0.897 \\
2D & 0.995 & 0.825 & 0.948 \\
Volumetric & 0.990 & 0.997 \\
\hline
\end{tabular}

${ }^{a}$ Assessed with the Pearson correlation coefficient.

${ }^{\mathrm{b}}$ Manual and CAV analysis.

Table 5: Interobserver agreement ${ }^{a}$ of 1D, 2D, and volumetric ${ }^{b}$ measurements

\begin{tabular}{lccc}
\hline & \multirow{2}{*}{$\begin{array}{c}\text { Interobserver } \kappa \\
\text { Correlation }\end{array}$} & \multicolumn{2}{c}{$95 \% \mathrm{Cl}$} \\
\cline { 3 - 4 } & 0.760 & Lower Limit & Upper Limit \\
\hline ID & 0.851 & 0.555 & 0.966 \\
2D & 0.948 & 0.882 & 1.000 \\
Volumetric & & \multicolumn{2}{c}{} \\
\hline
\end{tabular}

${ }^{a}$ Assessed with the $\kappa$ coefficient.

${ }^{\mathrm{b}}$ Manual and CAV analysis.

assessment has several unique advantages over linear-based techniques, including its ability to objectively accommodate the irregular and eccentric growth of GBMs, exclude cystic and necrotic components, and account for changes in the shape of the postoperative resection cavity. ${ }^{10,29}$ given the inherent morphologic features. Such variability has been observed within $2 \mathrm{D}$ measurements by the same reader with a reported $14 \%$ false-positive rate for diagnosis of disease progression in otherwise stable disease. $^{25}$

In this study, we subjectively observed a greater discordance between extrapolated volumes of linear-based techniques and volumetric assessment for tumors with increased eccentricity and necrotic changes. Specifically, linear measurements obtained for eccentric tumors would often be overestimated. Additionally, linear measurements obtained for cystic and necrotic tumors would often be overestimated. We also observed that extrapolated volumes for linearbased measurements of rounded nodular lesions were closer to the volumetric assessment. For reasons described previously, linear-based measurements are problematic for GBM assessment, given that these tumors are not typically solid ellipsoid lesions but display complex morphology. Such inconsistencies and potential inaccuracies may result in classifying effective treatments as ineffective or ineffective treatments as effective, which underscores the need for a reliable, reproducible, standard process of accurately determining tumor volume. ${ }^{6}$ With respect to use of computer-aided tools, researchers in body imaging have previously demonstrated improved accuracy over linear-based measurements by using a similar automated technique in the evaluation of lung, liver, and lymph node tumors. ${ }^{6}$

When one interprets the results of our study, several limitations should be kept in mind. First, this study is retrospective in design and subject to its limitations. Second, we have limited our analysis to enhancing contrast-enhancing tissue, which only represents increased blood-brain barrier permeability. While this may represent enhancing tumor, other possibilities may include inflammation, subacute ischemia, and so forth. However, there is no clear consensus on how to best assess the extent of residual and/or recurrent disease, which tends to default to the standard 1D or 2D measurement techniques, which are suboptimal. Additionally, there is no clear objective consensus on how to assess nonenhancing disease. Last, several 
patients were imaged within 12 weeks following resection, which is within the timeframe of pseudoprogression. It would have been optimal to repeat those studies to ensure that enhancement detected by CAV was indeed disease progression. ${ }^{11}$ However, histopathologic confirmation of recurrent disease was available for all except 1 patient imaged within the timeframe of pseudoprogression. Furthermore, the primary goal of this study was to assess the volumetric technique in comparison with other methods.

\section{CONCLUSIONS}

We have demonstrated the feasibility of a semiautomated segmentation technique to determine recurrent and/or residual tumor volume in patients with GBM, and this more reliable, reproducible, and significantly faster volumetric assessment of enhancing tumor burden has implications for the monitoring of disease progression and a potential role in therapy and novel treatment trials. Future studies should address patient outcomes with volumetric disease categorization, assess reliability among a larger number of readers, and compare reliability among different CAV algorithms.

Disclosures: Andrew B. Lassman—UNRELATED: Consultancy: Genentech, Merck Sharp \& Dohme, GlaxoSmithKline, Abbott, Ludwig Institute for Cancer Research, Kyowa Hakko Kirin Pharma, Novartis, RadMD, Roche, CampusBio, Qatar National Research Fund, Italian Association for Cancer Research, Grants/Grants Pending: Abbott,* Aeterna Zentaris, * Agenus, ${ }^{*}$ Amgen, ${ }^{*}$ Bayer and Onyx, ${ }^{*}$ Boehringer Ingelheim, ${ }^{*}$ Bristol-Myers Squibb, ${ }^{*}$ Celldex, ${ }^{\star}$ Genentech, ${ }^{\star}$ Keryx Biopharmaceuticals, ${ }^{*}$ Medimmune, ${ }^{*}$ Merck Sharp \& Dohme, ${ }^{*}$ Millenium, ${ }^{*}$ Northwest Biotherapeutics, ${ }^{*}$ Novartis, ${ }^{*}$ Pfizer, ${ }^{*}$ Plexxicon, ${ }^{*}$ Roche, ${ }^{*}$ Sigma Tau, ${ }^{*}$ Theorum Clinical Research, ${ }^{*}$ Payment for Lectures (including service on Speakers Bureaus): Merck Sharp \& Dohme, Omniprex, American College of Radiation Oncology, Travel/Accommodations/Meeting Expenses Unrelated to Activities Listed: American Academy of Neurology, United Council for Neurologic Subspecialties. Binsheng Zhao-UNRELATED: Patents (planned, pending, or issued): A patent for our in-house segmentation algorithm is pending (no payment received). Christopher G. Filippi-UNRELATED: Consultancy: MRI Advisory Board for Guerbet LLC, Comments: I spent 1 day listening to lectures on an ionic macrocyclic contrast agent developed by this company and used primarily in Europe with no cases of nephrogenic systemic fibrosis to date. I offered advice to the company on whether such an agent would be beneficial in the United States. I received a \$2000 honorarium for the day, Grants/ Grants Pending: 10\% effort on R01CA161404 of Dr Jeffrey N. Bruce, neurosurgeon, Comments: Phase II trial on pigs with new chemotherapy for GBM (I am responsible for the MR imaging component). *Money paid to the institution.

\section{REFERENCES}

1. Stupp R, Hegi ME, Mason WP, et al. Effects of radiotherapy with concomitant and adjuvant temozolomide versus radiotherapy alone on survival in glioblastoma in a randomised phase III study: 5-year analysis of the EORTC-NCIC trial. Lancet Oncol 2009;10:459-66

2. Reardon DA, Galanis E, DeGroot JF, et al. Clinical trial end points for high-grade glioma: the evolving landscape. Neuro Oncol 2011;13:353-61

3. van den Bent MJ, Vogelbaum MA, Wen PY, et al. End point assessment in gliomas: novel treatments limit usefulness of classical Macdonald's criteria. J Clin Oncol 2009;27:2905-08

4. Iliadis G, Kotoula V, Chatzisotiriou A, et al. Volumetric and MGMT parameters in glioblastoma patients: survival analysis. BMC Cancer 2012;12:3

5. Dempsey MF, Condon BR, Hadley DM. Measurement of tumor "size" in recurrent malignant glioma: 1D, 2D, or 3D? AJNR Am J Neuroradiol 2005;26:770-76

6. Schwartz LH, Ginsberg MS, DeCorato D, et al. Evaluation of tumor measurements in oncology: use of film-based and electronic techniques. J Clin Oncol 2000;18:2179-84

7. Therasse P, Arbuck SG, Eisenhauer EA, et al. New guidelines to evaluate the response to treatment in solid tumors: European Organization for Research and Treatment of Cancer, National Cancer Institute of the United States, National Cancer Institute of Canada. J Natl Cancer Inst 2000;92:205-16
8. Macdonald DR, Cascino TL, Schold SC Jr, et al. Response criteria for phase II studies of supratentorial malignant glioma. J Clin Oncol 1990;8:1277-80

9. Eisenhauer EA, Therasse P, Bogaerts J, et al. New response evaluation criteria in solid tumours: revised RECIST guideline (version 1.1). Eur J Cancer 2009; 45:228-47

10. Henson JW, Ulmer S, Harris GJ. Brain tumor imaging in clinical trials. AJNR Am J Neuroradiol 2008;29:419-24

11. Wen PY, Macdonald DR, Reardon DA, et al. Updated response assessment criteria for high-grade gliomas: response assessment in neuro-oncology working group. J Clin Oncol 2010;28:1963-72

12. Welsh JL, Bodeker K, Fallon E, et al. Comparison of response evaluation criteria in solid tumors with volumetric measurements for estimation of tumor burden in pancreatic adenocarcinoma and hepatocellular carcinoma. Am J Surg 2012;204:580-85

13. Zhao B, Oxnard GR, Moskowitz CS, et al. A pilot study of volume measurement as a method of tumor response evaluation to aid biomarker development. Clin Cancer Res 2010;16:4647-53

14. Mozley PD, Bendtsen C, Zhao B, et al. Measurement of tumor volumes improves RECIST-based response assessments in advanced lung cancer. Transl Oncol 2012;5:19-25

15. Kanaly CW, Ding D, Mehta AI, et al. A novel method for volumetric MRI response assessment of enhancing brain tumors. PLoS One 2011;6:e16031

16. Egger J, Kapur T, Fedorov A, et al. GBM volumetry using the 3D Slicer medical image computing platform. Sci Rep 2013;3:1364

17. Zhu Y, Young GS, Xue Z, et al. Semi-automatic segmentation software for quantitative clinical brain glioblastoma evaluation. Acad Radiol 2012;19:977-85

18. Computer-aided tumor segmentation using local region-based active contours. Columbia University Invention Report IR \#2906

19. Methods and Systems for Segmentation of Organs and Tumors and Objects. Patent (pending) PCT/US13/36166. April, 2013

20. Zaretsky J, Guo X, Fu J, et al. Novel radiologic features to predict hepatocellular carcinoma recurrence after liver transplantation: a pilot study. In: 62nd Annual Meeting of the American Association for the Study of Liver diseases (AASLD), San Francisco, California. Nov 3-8, 2011

21. Abou-Alfa GK, Zhao B, Capanu M, et al. Effects of Radiologic Tumor Response of Anti-Glypican 3 GC33 and Multi Tyrosine Kinases Inhibitor Sorafenib in Hepatocellular Carcinoma. In: 24th EORTCNCI-AACR Symposium on Molecular Targets and Cancer Therapeutics. Dublin, Ireland. November 6-9, 2012

22. Leinwand J, Zhao B, Guo X, et al. Quantitative CT peritoneography in malignant peritoneal mesothelioma patients receiving intraperitoneal chemotherapy. Ann Surg Oncol 2013 May 24. [Epub ahead of print]

23. Sorensen AG, Patel S, Harmath C, et al. Comparison of diameter and perimeter methods for tumor volume calculation. J Clin Oncol 2001;19:551-57

24. Provenzale JM, Mancini MC. Assessment of intra-observer variability in measurement of high-grade brain tumors. J Neurooncol 2012;108:477-83

25. Provenzale JM, Ison C, Delong D. Bidimensional measurements in brain tumors: assessment of interobserver variability. AJR Am J Roentgenol 2009;193:W515-22

26. Sorensen AG, Batchelor TT, Wen PY, et al. Response criteria for glioma. Nat Clin Pract Oncol 2008;5:634-44

27. Keles GE, Lamborn KR, Chang SM, et al. Volume of residual disease as a predictor of outcome in adult patients with recurrent supratentorial glioblastomas multiforme who are undergoing chemotherapy. J Neurosurg 2004;100:41-46

28. Lacroix M, Abi-Said D, Fourney DR, et al. A multivariate analysis of 416 patients with glioblastoma multiforme: prognosis, extent of resection, and survival. J Neurosurg 2001;95:190-98

29. Warren KE, Patronas N, Aikin AA, et al. Comparison of one-, two-, and three-dimensional measurements of childhood brain tumors. J Natl Cancer Inst 2001;93:1401-05

30. Gladwish A, Koh ES, Hoisak J, et al. Evaluation of early imaging response criteria in glioblastoma multiforme. Radiat Oncol 2011;6:121 